\title{
ASPECTOS DO VOCABULÁRIO E SEMÂNTICA DO PORTUGUÊS ARCAICO
}

Sob este título ocupamo-nos aqui de alguns aspectos dos vocábulos que constituem as crónicas em português dos Portugaliae Monumenta Historica (vol. Scriptores, publicado em Lisboa, em 1856, por Alexandre Herculano). Todos os problemas relacionados com essa edição, principalmente do ponto de vista diplomático e paleográfico, se encontram por nós estudados em extenso artigo da Revue des Langues Romanes, tomo LXXVII (1967), Les Chroniques Portugaises des Portugaliae Monumenta Historica. A ortografia de todos os exemplos é a que apurámos, nos textos que serviram de fonte à crónicas, ser a mais fidedigna, e nem sempre a ocorrente nos Scriptores, a que, no entanto, para comodidade do leitor, nos reportamos, indicando a página, coluna e linha onde os mesmos se acham, muitas vezes em leitura menos exacta.

Primeiramente apresentamos o elenco dos LATINISMOS (1.), começando pelos ortográficos e fonéticos (a). Exemplos em que um $g$ esconde a pronúncia $i$ : Regnou $(22, \mathrm{~B}, 53)$, Regno $(31, \mathrm{~B}, 45)$; vocábulos onde um $c$ está nas mesmas condições: octubro $(29, \mathrm{~A}, 30)$, despecto $(24, \mathrm{~B}, 3)$, perfecto $(78, \mathrm{~B}, 55)$, electo $(24, \mathrm{~B}, 27)$; em actoridade $(25, \mathrm{~A}, 7)$, o $c$ já não se pronunciava, mas podia representar o $u$ etimológico como ele (auctoritate->autoridade); em juventute $(77, \mathrm{~B}, 21)$, o segundo $t$ mascara o $d$ a que dera origem (juventude) e em Redemptor $(24, \mathrm{~B}, 57)$ o $p$ já devia ser mudo, como hoje (redentor). Como latinismos morfológicos (b) estão os superlativos eruditos inuictissimo e christianissimo (407,B,19, no título da crónica) e santissima $(76, \mathrm{~B}, 56)$.

Passando aos latinismos semânticos e lexicais (c), citem-se costantinopoly $(76, \mathrm{~A}, 10)$, termo latino proveniente do grego, pelo actual Constantinopla; genesy $(78, \mathrm{~B}, 58)$, por Génesis; adliterom $(78, \mathrm{~B}, 59)$, bibliónimo arcaico, isto é, Vulgata; color $(28, \mathrm{~A}, 11)$, usado até tarde, ao lado de cor, que dele provém; persoalmente $(25, \mathrm{~B}, 21)$, cuja forma vernácula é pessoalmente (o latim $r s$, precedido de vogal, deu ss em português, como no arcaico osso, de ursu-, ou os hodiernos pêssego, de persicu-, e avesso, de adversu-); sobrinho (22,B,23), latinismo semântico quando no sentido de 'primo', como no local citado.

O latinismo sintáctico (d) mais importante é o emprego de nem com o sentido arcaico de $e$ (copulativo en vez de disjunctivo). Eis o passo em que occore: "Eeste era o mais esfforçato caualleiro em armas Eem força que auija em espanha, nẽ deque os mouros mayor medo auijan" $(29, \mathrm{~B}, 5)$.

PSEUDOLATINISMOS (2.) ortográficos, devidos a ultracorrecção, temos sobçessor $(23, \mathrm{~B}, 9)$, correspondente ao actual sucessor, causado dela consciência da composição da palavra com o prefixo sub-, que deu so(b); supito $(417, \mathrm{~A}, 30)$, cujo $p$ 
se deve a regressão por pseudo-eruditismo (súbito já tinha $\mathrm{b}$ no étimo); dãpno $(27, A, 23)$, cujo $p$ não tem valor fonético, não passa também de mero pseudoeruditismo (cf. Edwin Williams, From Latin to Portuguese, ${ }^{*} 26,1$ ), e dapno $(27, \mathrm{~B}, 52)$, que poderia ter vindo do anterior, por desnasalação $(\tilde{a}-n>a-n)$, mas onde é mais provável que o escriba se tenha esquecido do til; escrepuer $(78, \mathrm{~B}, 47)$ ou escripuer $(78, \mathrm{~B}, 43)$, cujo $p$, além de mudo, é pseudo-erudito, por influência do part. pass. escri(p)to (cf. E. W., From Latin, *28,2); e cluz (30,B,56), por cruz (o escriba, pouco sabedor da língua latina, pensava certamente que a $\mathrm{cr}$ - inicial em português correspondia sempre $\mathrm{cl}$ - em latim).

Prosseguimos o presente trabalho com o rol de todas as CONVERGENTES (3.) encontradas nos textos das crónicas:

a: 1. artigo $(22, \mathrm{~A}, 17)$, pron. pess. $(24, \mathrm{~B}, 1)$ e pron. dem. $(24, \mathrm{~A}, 46)<i l l a ̆-$, por próclise, através da forma la, depois de vogal: 2. prep. $(22, \mathrm{~B}, 28)<a d$; $c a$ : 1 . conj. causal $(25, \mathrm{~A}, 4)<q u i a ; 2$. conj. compar. $(29, \mathrm{~B}, 66)<q u a m$; delo: $1 .<>$ disso $(410, \mathrm{~B}, 26)<$ de + elo $<$ illud; $2 .<>$ desde) o (de dês $+l o$ );

dom: 1. título $(22, \mathrm{~A}, 19)<$ provençal don < dominu-; 2 . dádiva $(d \tilde{o} \tilde{e} e s, 78, \mathrm{~B}, 14)$

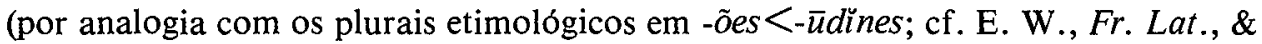
$123,4, \mathrm{~A})$;

era: 1 . subst $(22, \mathrm{~A}, 19)<>$ época: 1 . imperf. do v. $\operatorname{ser}(26, \mathrm{~A}, 4)$;

leito: 1. cama $(78, \mathrm{~A}, 22) ; 2$. eleito $<\bar{e} l e \bar{c} t u-(31, \mathrm{~B}, 38)$;

mão: 1 . subst. (mãão, 25,A,55); 2 . adj. $<>$ máo, arc., màu (mããos [sic] conselheiros, 31,B,35)<malu-; a nasalidade pode ter-se estendido ao $a$, mas é mais natural tratar-se de distracção do copista, habituado a grafar assim a parte do corpo humano (1.);

marta: 1. (Rey das martas, 31,B,29); 2. Santa Marta (77,A,71).

no: 1 . redução de nom, devida à próclise $(29, \mathrm{~A}, 66$ e $28, \mathrm{~A}, 58) ; 2$. variante do artigo $(75, \mathrm{~B}, 45)$ e pron. $(30, \mathrm{~A}, 52): 3$. contr. da prep. em e do art. arc. lo $(22, \mathrm{~A}, 40) ; 4$. contr. da prep. em e do pron. dem. lo (no(s) da vila, 418,A,60);

nos: 1. pronome pessoal da $1 .^{\mathrm{a}}$ pessoa $(26, \mathrm{~B}, 23) ; 2$. variante do artigo $(75, \mathrm{~B}, 38) ; 3$. contracção da preposição em e do pronome demonstrativo los $(418, \mathrm{~A}, 60)$;

quedo: 1. adj. (quedos, 419,B,46); 2. v. quedar (30,B,5);

rogo: 1 . subst. $(26, \mathrm{~A}, 53) ; 2$. v. rogar $(26, \mathrm{~B}, 48)$;

são: 1. santo $(418, \mathrm{~B}, 1)$; sadio (sãão, $407, \mathrm{~B}, 37) ; 3$. v. ser, $3^{\mathrm{a}}$ p. pl. do pres. do ind. $<$ sunt $(417, \mathrm{~B}, 37)$;

see: 1 . subst. (sees cathedraaes, $25, \mathrm{~A}, 5) ; 2$. v. seer (ssee aadestra, $28, \mathrm{~A}, 55)<$ sèdet; $50)$;

SEI: 1. v. saber (bẽ sey, 412,B,26); 2. v. ser<sede (ssey companheiro, 26,A, seja: $1.1^{\mathrm{a}}$ p. sing. conjuntivo pres. $(77, \mathrm{~B}, 19) ; 2.3^{\mathrm{a}}$ p. sing. conj. pres. (sseia, $28, \mathrm{~B}, 7)$;

som: 1 . adj. <sõlu- $(411, \mathrm{~A}, 26) ; 2$. v. ser, $1^{\mathrm{a}}$ p. sing. pres. ind. $<$ sum (eu soo, $30, \mathrm{~A}, 16)$;

som: 1. v. ser, $1^{\text {a }}$ p. sing. pr. ind. $<\operatorname{sum}\left(\right.$ eu soom, 26,B,49); 2. id., $3^{\mathrm{a}}$ p. pl. <sunt $(22, \mathrm{~A}, 33) ; 3$. subst. (o som deles, 417,A,51); 
veer: 1 . ver < vidēre $(77, \mathrm{~A}, 12) ; 2$. vier < vēněrit, com deslocação do acento (ueher, 412,B,7).

Damos finalmente a extensa lista de DIVERGENTES (4.) colhida nas crónicas: $\grave{a}(75, \mathrm{~B}, 30)$ e aa $(22, \mathrm{~A}, 54)<\breve{a}(d \breve{l}) l a-$, por próclise; aaquel $(410, \mathrm{~B}, 15)$ e aquele $(419, \mathrm{~A}, 1)$, por àquele (prep. $a+$ pron. dem. aquel(e)<*acc(u)ille; cf. E. Bourciez e Grandgent, apud Antenor Nascentes, Dicionário Etimológico da Língua Portuguesa, I, s. v.); abito $(76, \mathrm{~B}, 69)$ e auito $(414, \mathrm{~A}, 34)<$ habïtu-; acaecer $($ Acaeceu, 409,B, $59)$ e aquecer $(417, \mathrm{~B}, 39)<$ accadescère (cf. José Pedro Machado, Dicionário Etimológico da Língua Portuguesa, $1^{\mathrm{a}}$ ed., p. 42$)$; aceca $(76, \mathrm{~B}, 73)$ e açerqua $(25, \mathrm{~B}, 63)<$ ad circa (a síncope do $r$ da $1^{\text {a }}$ forma explica-se talvez por próclise); [acender] (acendam, $72, \mathrm{~B}, 29)$ e [ascender] (asçendeo, 28, A,55)<ascendĕre; açeriago (76, B,76), açeriagoo $(77, \mathrm{~A}, 65)$, arcediago $(24, \mathrm{~B}, 11)$ e arçeriagoo $(77, \mathrm{~A}, 4)<$ archidlacŏnŭ- $<\mathrm{gr}$.

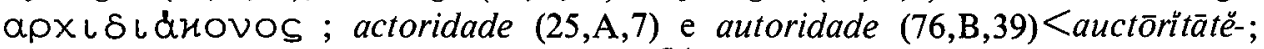
[aficado] (aficada, 408,A, 14), de aficar<*fïgícāre, frequentativo de figere (cf. J. P. M., D.E.). e [afincado] (afi(n)cados, 418,A,24), de afincar<*fin(gü)cāre, por figìcăre (c. J. P. M., D.E.); agoua $(25, \mathrm{~B}, 58)$ (é difícil explicar a ditongação do -u-em -ou-; talvez deva admitir-se como intermediária a forma áugua (augoa), de cujo primeiro ditongo resultaria, por assimilação a distância, -ou- na segunda síl.), agoa $(29, \mathrm{~B}, 72)<\breve{a q u a ̆-~ e ~ a u g o a ~}(29, \mathrm{~A}, 20)$, cruzamento de água com a forma pop. e arc. auga; alcaçar $(419, \mathrm{~B}, 6)$ e alcaçer $(30, \mathrm{~B}, 1)<$ ár. al-qaÇr, 'palácio', arabização do lat.

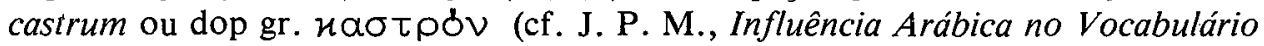

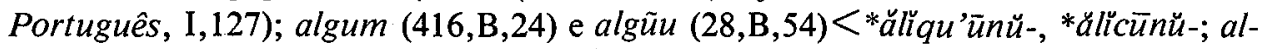
guns $(417, \mathrm{~A}, 26)$ e algũus $(76, \mathrm{~A}, 40) *$ ăliqu'ūunōs, *ălĭcūnōs; allo $(22, \mathrm{~B}, 54)$ e allos $(27, \mathrm{~A}, 68)$, do $1^{\circ}$ com $s$ final analógico com o de outros adv. (aló< $\ddot{a} d+\ddot{l} l l \bar{c} c$; cf. Meyer-Lübke, Romanisches Etymologisches Wörterbuch, $\left.\mathrm{n}^{\circ} 4270\right)$; ante $(26, \mathrm{~A}, 44)$, de antĕ, e antes (?) $(416, \mathrm{~A}, 32)$; antre $(29, \mathrm{~A}, 34)$ e entre $(417, \mathrm{~B}, 20)<$ inter; aos $(24, \mathrm{~A}, 36)$ e $[o ̀ s]$ (os, 29, $\mathrm{B}, 41)$; aquell $(29, \mathrm{~A}, 15)$, através de aquelle $(26, \mathrm{~A}$, $45)<* a c c(u)$ llle (cf. E. Williams, From Latin, * 145,1); aRancado $(26, \mathrm{~B}, 31)$ e aRincado (29, B, 70), de arrancar e arrincar lat. med. (ar)rancare eruncare, sob a influência do germ. rank e do gót. *wrankjan, 'puxar retorcendo' (cf. Cornu, Portugiesische Sprache, $\S 92$, García de Diego, Contribución al Diccionario Hispánico Etimológico, $\mathrm{n}^{\circ} 212$, M.-L., REW, $\mathrm{n}^{\circ} 7044$, J. P. M., D.E., s. v. arrancar; para Adolfo Coelho, Dicionario Manual Etymologico da Lingua Portugueza, arrancar vem do lat. radicari com o pref. $a$, preservando a nasalização do $a$ o $c$ do abrandamento que se nota em arraigar; cf. ainda Cortesão, Subsídios para um Diccionario Completo (Històrico-Eymológico) da Língua Portuguêsa, A. Nascentes, D.E., I, s. v. arrancar); arrabalde $(25, \mathrm{~B}, 70)$ e arraualde(s) $(25, \mathrm{~B}, 55)<$ ár. araba $\bar{D}$, pl. de $a r$-rabD, 'subúrbios, arredores', ou, mais, provavelmente, desta pal. tornado ar-rabáD, por deslocação do acento na Hispânia (cf. J.P.M., Infl. Ar., s.v. Arrabalde); auedes $(410, \mathrm{~A}, 32)$ e aueis $(418, \mathrm{~B}, 23)$ além de ouuerom $(26, \mathrm{~B}, 28)$ e ouueram $(25, \mathrm{~A}, 49) ; a z$ $(26, \mathrm{~B}, 30)$ e azaa (s) $(29, \mathrm{~B}, 67)<$ acie- .

Beençam $(26, \mathrm{~A}, 77)$, beençom $(77, \mathrm{~B}, 62)$ e béç̧om $(25, \mathrm{~A} 46)$ ou bençõ $(409, \mathrm{~A}$, $13)<$ benedictiōne-; beijar $(28, \mathrm{~A}, 35)$, e bejar $(78, \mathrm{~B}, 35)<$ basiare; beenzer $(411, \mathrm{~A}, 8)$ e benzer $(410, \mathrm{~B}, 52$, no tít. do cap. VIII)<benedicĕre; boo $(30, \mathrm{~B}, 62)$ e bõo $(27, \mathrm{~A}$, 
$20)<$ bonu-; chaman $(25, \mathrm{~A}, 70)$ e chamom $(31, \mathrm{~A}, 10)$; chamaram $(24, \mathrm{~A}, 49)$ e chamarõ $(24, \mathrm{~A}, 46)$; çinquo $(30, \mathrm{~A}, 39)$ e çinqui $(22, \mathrm{~B}, 56)<$ quinque, através do lat. pop. cinque; claro (clara, $75, \mathrm{~B}, 47)$, e craro $(417, \mathrm{~A}, 10)$ claru-; clastra $(30, \mathrm{~A}, 47)$, clasta $(76, \mathrm{~B}, 13)$, claustra $(24, \mathrm{~B}, 14)$ e crasta $(28, \mathrm{~A}, 4))<$ claustra; clerizia $(76, \mathrm{~A}, 28)<*$ clericia-, de clèricu- (cf. J.P.M., D.E., s.v. clero ${ }^{1}$ ) e crelizia $(408, A, 34)$, de primeiro termo, através de ${ }^{*}$ crerizia, por diss. do $2^{\circ} \mathrm{r}$; cluz $(30, \mathrm{~B}, 56)$, pseudolatinismo ortogr. (v. mais acima) e cruz $(24, \mathrm{~A}, 73)$; co $(29, \mathrm{~B}, 51)$, redução de com $(22, \mathrm{~A}, 36)$; coitelo $(410, \mathrm{~B}, 59)$ e cuitelo $(410, \mathrm{~B}, 62)<$ cultellu-; come $(441, \mathrm{~A}, 33)<$ quomo(do) e et para alguns etimologistas (cf. Bruno Migliorini, Prontuario Etimologico della Lingua Italiana, ital. come) e como $(24, \mathrm{~A}, 72)$; começa $(407, \mathrm{~B}, 39)$ e compeça $(29, \mathrm{~B}, 7)$ (compeçar é cruzamento de empeçar<*impeditiare e *començar *cominitiare; cf. Gunnar Tilander, Notas Etimológicas, p. 7); começo $(22, \mathrm{~A}, 18)$ e compeço $(31, \mathrm{~B}, 22)$, der. regressivo de compeçar; comjgo $(26, \mathrm{~A}, 63)$ e cõ migo $(410, \mathrm{~B}$, 21)<cum + mécu migo, através de *micu-, por infl. anal. do $i$ de $m i$ (cf. Huber, Altportugiesisches Elementarbuch, $\$ 89,2)$; companha $(413, \mathrm{~B}, 47)$ e companhia $(75, \mathrm{~B}$, $16)$; condição $(420, \mathrm{~B}, 8)$ e condiçom $(27, \mathrm{~A}, 38)<$ condicione-; conego $(414, \mathrm{~A}, 10$, no tít. do cap. XV), coonego $(25, \mathrm{~A}, 37)$ e coonigo $(414, \mathrm{~B}, 13)<$ cănŏnĭcu- (a última $\mathrm{f}$. pressupõe *caónigo, de que provém por assimil. do a ao o); [confirmar] (confirmo, 414,A,4) e [cunfirmar] (cumfirmou, 77,A,22)<confirmáre; conheçer, 414,A,4) e [cunfirmar] (cumfirmou, 77,A,22)<confirmāre; conheçer $(26, \mathrm{~B}, 70)$, [conhicer] (conhiçia, 31,A,20) e [conhocer] (conhoçamos, 411,B,5)<cognoscëre, as duas primeiras por dissimil. da última; consentir $(414, \mathrm{~B}, 3)$ e consintir $(27, \mathrm{~B}, 3)<$ consentīre (o $i$ medial deve-se à anal. com o da $1^{\text {a }}$ pess. do sing. do pres. do ind. e à assimil. ao $i$ da desinência); considerar $(417, \mathrm{~A} 26)$ e consirar $(75, \mathrm{~B}, 19)<$ consīdĕrāre; coraçã $(29$,$\mathrm{B}, 23)$ e coraçõ $(28, \mathrm{~A}, 71)<*$ coratiōne-, deriv., substituto e sinónimo vulg de cor, através de corātum (cf. J.P.M., D.E., p. 515); Coronjqua $(416, \mathrm{~A}, 17)$ e cronjca $(25, \mathrm{~A}, 4)<c h r o ̈ n i ̆ c a$, pl. neutro, sendo a ${ }^{\mathrm{a}} \mathrm{f}$., tal como a frequente carónica, devida a suarabácti, por infl. da vibrante; creligo (creligos, $24, \mathrm{~B}, 34)$ e crerigo $(28, \mathrm{~A}, 11)<$ clēricu-; cujya, isto é cuija $(78, \mathrm{~B}, 9)$ e cuja $(76, \mathrm{~B}, 2)$, fem. de cujo <cujju-.

Dãpno $(27, \mathrm{~A}, 23)$, dapno $(27, \mathrm{~B}, 52)$ e dano $(417, \mathrm{~B}, 39)<$ damnu-;dessi, igual a desi $(407, \mathrm{~B}, 36)$, e disy $(29, \mathrm{~B}, 9)$, de des $<$ de ex ibi; dell $(29, \mathrm{~A}, 50)$ e dele $(418, \mathrm{~A}, 63)$, de de +ele <ille; depois $(24, \mathrm{~A}, 5)$, depos $(30, \mathrm{~B}, 24)$, despos $(77, \mathrm{~A}, 6)$ e despovs $(25, \mathrm{~B}$, 44), h de ex post(e), os dois últimos (cf. J.P.M., D.E., p. 757 B); [destrebuir] (destrebuy, 412,A,20) e destribuir $(412, \mathrm{~A}, 52)<$ distribüere); deuação $(418, \mathrm{~B}, 16) \mathrm{e}$ deuaçõ $(408, \mathrm{~A}, 35)<$ devotiōne-; deserom $(76, \mathrm{~A}, 29)$ e disserom $(26, \mathrm{~A}, 71)<$ dixerünt; dezia $(75, \mathrm{~B}, 36)$ e dizia $(408, \mathrm{~B}, 49)<$ dicēe(b)at; dignjdade $(25, \mathrm{~A}, 17)$ e dinjdade $(25, \mathrm{~A}, 64)<$ dignitāte-; deujson $(27, \mathrm{~B}, 22)$ e diuison $(411, \mathrm{~B}, 37)<*$ divisaut; door $(26, \mathrm{~A}, 41)$ e dor $(418, \mathrm{~B}, 5)<$ dolöre-; ell $(28, \mathrm{~A}, 8)$ e elle $(75, \mathrm{~B}, 51)<$ ille; ellecto $(24, \mathrm{~B}, 27)$, emleito $(78, \mathrm{~B}, 31)$ e leito (leitos, $31, \mathrm{~B}, 38(<\bar{e} l \bar{e} c t u$-, apresentando a últ. $\mathrm{f}$. a aférese do $e$-átono inicial e constituindo síl. só por si, o que é frequente; ẽmiigo(s) (407,A,26), emijgu(s) $(76, \mathrm{~A}, 40)$ e Jmmigo(s) $(25, \mathrm{~A}, 12)<$ inimīcu-; êfermidade $(410, \mathrm{~A}, 36)$, enfirmjdade $(76, \mathrm{~A}, 28)$ e Jnfirmjdade $(77, \mathrm{~A}, 67)<$ infirmitāte-; emperador $(25, \mathrm{~A}, 69)$ e Jmperàdor $(24, \mathrm{~A}, 19)<$ imperatōrě̀-; Emsinar $(76, \mathrm{~A}, 4)$ e insynar (76,A,6)<*insignāre, em vez de insignīre (cf. J.P.M., D.E., p. 1968, s.v. senho); entenção $(409, \mathrm{~A}, 46)$ no tít. do cap. III) e emtẽeçõ $(76, \mathrm{~B}, 24)<$ intentiōne-; 
entam $(24, \mathrm{~A}, 59)$ e entom $(22, \mathrm{~B}, 24)$ in + tunc; entonçe $(30, \mathrm{~A}, 46)$, ẽntonçes, cujo -e é analógico $(30, \mathrm{~A}, 57)$, estonçes cujo -s também é anal. com os adv. em -s (cf. Huber, Altport., $\S 297)(29, \mathrm{~B}, 48)$, e Estonçe $(30, \mathrm{~A}, 57)$ (as f. sem is vêm de *intünce (cf. M.-L., $R E W, \mathrm{n}^{\circ} 4518$ ), a $1^{\mathrm{a}}$, e talvez de *extŭnce, por in ou ex +tunc, a $2^{\mathrm{a}}$, segundo José Joaquim Nunes, Crestomatia Arcaica, §156); emviedes $(30, \mathrm{~B}, 39)$ e emvijees (78,B,10-12); escrepuer $(78, \mathrm{~B}, 47)$ e escripuer $(78, \mathrm{~B}, 43)<$ scribĕre; escriptura(s) $(22, \mathrm{~A}, 24)$ e scretura $(407, \mathrm{~A}, 39)<$ scriptūra; escreueo $(76, \mathrm{~A}, 11)$ e escripueo $(78, \mathrm{~B}$, 39), f. regulares ou analógicas; esmaginaua (78,B,53-54) e maginaua (76,A, $20)<$ (i)maginābat, com o pref. expletivo es- na $1^{\text {a }}$ forma; espargeron $(409, \mathrm{~B}, 52) \mathrm{e}$ espargirom $(418, \mathrm{~B}, 16)$; esperito $(78, \mathrm{~A}, 41)$, espiritu $(76, \mathrm{~A}, 45)$, e esprito $(31, \mathrm{~A}, 3)<$ spiritu-; esporas $(418, \mathrm{~B}, 62)$ e esperoras $(31, \mathrm{~A}, 34)<$ gót. ${ }^{*}$ spaura, ${ }^{*}$ spora; spirital $(24, \mathrm{~B}, 40)$, espritall $(29, \mathrm{~A}, 70)$ e hospital $(25, \mathrm{~A}, 52)$, os dois primeiros vocábulos produto do cruzamento de espírito ou esprito com o último; [estrangeiro] (estrangeiras, $409, \mathrm{~B}, 22$, no tít do cap. V) e [estranheiro] (estranheiros, 411,B,30), o segundo vernáculo <*extrāniariu-, de extrānëus; enxardar $(27, \mathrm{~A}, 26)$ e exerdar (exerdaste, 26,B,47)< exhērēdāre (cf. J.P.M., D.E., P. 1165 B), o primeiro com nasalização espontânea e a vog. da síl. medial alterada por infl. do $r$.

Fez $(22, \mathrm{~B}, 48)$ e feze $(24, \mathrm{~A}, 29)<$ fëcit; fezesem $((23, \mathrm{~B}, 10)$ e fizesem $(419, \mathrm{~B}$, 56) fécissent; fezese $(25, \mathrm{~B}, 39)$ e fizese $(416, \mathrm{~B}, 25)<$ fēcisset; fezerom $\left(23\right.$, no tít. da $2^{\mathrm{a}}$ crón. breve) e fizeron (?) $(416, \mathrm{~B}, 30)$; fezera $(27, \mathrm{~B}, 2)$ e fizera $(416, \mathrm{~A}, 39)<$ fécĕrat, com deslocação, do acento; ffarey $(27, \mathrm{~A}, 33)$ e ffazer (te) ey $(26, \mathrm{~A}, 62)<*$ facer'aio; ffe $(28, \mathrm{~A}, 26)$ e fee $(418, \mathrm{~A}, 25)<$ fide-; foy se $(25, \mathrm{~A}, 51)$ e fosse, isto é fo[i]se $(26, \mathrm{~B}, 72)$, f. anal. com fosse, por confusão do pron.reflexo com a terminação -sse, erro muito vulgar ainda hoje; fora $(25, \mathrm{~A}, 67)$ e foras $(?)(416, \mathrm{~A}, 37)<$ foras; ffortellez $(29, \mathrm{~A}, 25)$ e ffortelleza(s) $(26, \mathrm{~B}, 65)<$ provençal fortaleza, der. de förtis (cf. M.-L., $\left.R E W, \mathrm{n}^{\circ} 3457\right)$; frade $(413, \mathrm{~A}, 40$ no tít. do cap. XIII), fraire(s) $(78, \mathrm{~A}, 19) \mathrm{e}$ Freyre(s) $(416, \mathrm{~B}, 14)$, os dois últimos do prov. fraire $<$ fratre- (cf. M.L., $R E W, \mathrm{n}^{\circ}$ $3485)$; flamento $(413, \mathrm{~A}, 40$, no tít. do cap. XIII) e framẽgo $(413, \mathrm{~A}, 51)<$ germ. flaming; gaanhado $(26, \mathrm{~A}, 34)$ e ganhado(s) $(416, \mathrm{~B}, 27)$, de guadaniatu-, de guadaniare<frâncico *waidanjan; gardador $(77, \mathrm{~B}, 42)$ e guardador $(410, \mathrm{~B}, 15)$, derivados de g(u)ardar<b.-lat. guardāre< $<$ germ. *wardōn, cf. J.P.M., D.E., s.v. Guardar; gardar $(75, \mathrm{~B}, 69)$ e [guardar] (guardaua, 409,B,13); grã $(407, \mathrm{~B}, 48)$, gram $(24, A, 10)$, grão $(419, \mathrm{~A}, 40)$, todas provenientes de grande $(24, \mathrm{~A}, 45)$, por próclise; home(s) $(407, \mathrm{~B}, 31)$ e homẽ $(407, \mathrm{~B}, 22)$ homine-; homes $(75, \mathrm{~B}, 16)$, homẽs $(75, \mathrm{~B}, 20)$ e homẽes $(24, \mathrm{~B}, 1)<$ homines; hora $(25, \mathrm{~A}, 27)$ e ora $(22, \mathrm{~A}, 19) ;$ Jfante $(22, \mathrm{~A}, 46)$ e Infante $(22, \mathrm{~B}, 15)<$ infante-; leixo $(26, \mathrm{~A}, 46)<$ laxo e lheixo $(29, \mathrm{~B}, 21)$, do primeiro, por assimil. causada pela palatal $x(l-x<l h-x)$; lhes $(408, \mathrm{~A}, 43)$ e lhis $(408, \mathrm{~B}, 41)$.

Madre $(25, \mathrm{~A}, 62)$ e mai $(76, \mathrm{~A}, 33)$, do anterior, por próclise; mayor $(24, \mathrm{~A}, 68)$, moor $(24, \mathrm{~B}, 59)$ e mor $(418, \mathrm{~B}, 7)<$ maiōrě-; mais $(25, \mathrm{~B}, 16)$ e mas $(25, \mathrm{~A}, 56)<$ magis; manham $(417, \mathrm{~B}, 8)$, manhãa $(28, \mathrm{~A}, 49)$, menham $(417, \mathrm{~A}, 9)$ e menhaa $(30, \mathrm{~A}, 45)<$ *maneāna-; mantou $(26, \mathrm{~B}, 63)$, em que houve extensão da nasalidade à vog. seg., e matou $(27, \mathrm{~B}, 54)$; maraujdẽs $(76, \mathrm{~B}, 11)$, marauidis $(29, \mathrm{~A}, 71)$ e maraujdījs $(77, \mathrm{~B}$, $1)<a ́ r . ~ m u r a ̄ b i T i n ~(u n) ; ~ m a r t e r ~(25, \mathrm{~B}, 10)$ e martir $(29, \mathrm{~A}, 57)<$ marty̆re-, do gr.

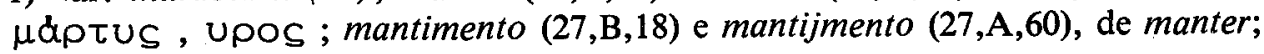


medo $(28, \mathrm{~A}, 23)$ e mendo $(30, \mathrm{~A}, 62)<m \bar{e} t u-; '$ memção $(416, \mathrm{~A} 17)$ e mençõ $(22, \mathrm{~A}, 26)<$ mentiōne-; merçe $(416, \mathrm{~A}, 38)$ e mercee $(27, \mathrm{~B}, 26)<$ mercēede-; $\mathrm{mi}$ $(412, \mathrm{~B}, 21)$, e $m \tilde{y}(26, \mathrm{~A}, 50)<\mathrm{mī}$, por mĭhi; mjnha $(26, \mathrm{~B}, 21)$ e mjhna $(75, \mathrm{~B}, 6)<$ mĕa-, através de *mea, mia e mīa (cf. E.W.Fr. Lat., $\$ \S 34,6$ e 66,1 B; a $1^{\text {a }}$ f. port., que é a actual, provém da arc. por desnasalação do $i)$; miraamollim $(25, \mathrm{~B}, 23)$, Mjramomolim $(419, \mathrm{~A}, 43)$ e Almjramomolim $(419, \mathrm{~A}, 50)<a ́ r . ~ m \bar{r}$ al-mūmnīn 'o príncipe-dos crentes' (cf. J.P.M., D.E., p. 168, B; a f. com al-, mais próxima do étimo, tem o art. def. ár. transposto para o princípo da pal., devido à anal. com a maioria das pal. de or. ár., começadas assim); mjzerado (30,A,68) e mjzzcrado $(28, \mathrm{~A}, 32)$, part. de mïscrar ou miscrar< $<$ misscüläre, frequentativo de miscēre ou der. do lat. tardio miscuere ou miscuare (cf. J.P.M., D.E., e A.N., D.E., I, s.v. mesclar, e M.-L., $\left.R E W, \mathrm{n}^{\circ} 5606\right)$; mester $(26, \mathrm{~B}, 23)$ e mjster $(419, \mathrm{~A}, 51)<$ ministëriu- (cf. J.P.M., D.E., p. 1510); mjssa $(28, \mathrm{~A}, 19)$ e missa $(28, \mathrm{~A}, 22)<m i s s a$ (na $2^{\mathrm{a}} \mathrm{f}$. houve nasalização do $i$ por infl. do $m$ ); moesteiro $(22, \mathrm{~A}, 53)$, mojsteiro $(416, \mathrm{~A}, 16)$ e $m o$ -

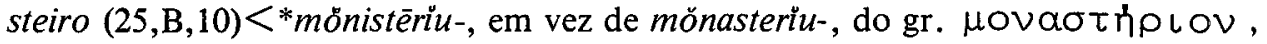
por anal. com baptisterium; cf. J.P.M., D.E., P. 1530 e Leite de Vasconcelos, Lições de Filologia Portuguesa, p. 91-92); muj $(25, \mathrm{~A}, 58)$ e mũj $(27, \mathrm{~B}, 10)$, de muito, por próclise; moimẽto $(410, \mathrm{~B}, 13)$ e muymento $(418, \mathrm{~B}, 19)<$ monimentu- ou molimentu- (cf. M.-L., REW, ${ }^{\circ} 5672$, que não dá a f. port.: a passagem de oi a $u i$ deve-se à metafonia; cf. J.N., Gramática Histórica da Língua Portuguesa, p. 157); mujto $(76, \mathrm{~B}, 36)$ e mũỹto $(24, \mathrm{~B}, 3)<$ multu-; multidooe $(25, \mathrm{~B}, 24)$ e moltidom $(25, \mathrm{~B}, 69)<$ multitudine- (na $1^{\mathrm{a}} \mathrm{f}$. o escriba esqueceu-se de assinalar a nasalidade da terminação).

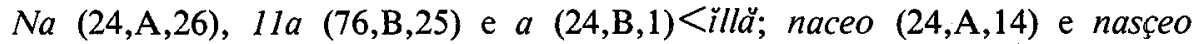
$(416, \mathrm{~A}, 10)$, de na(s)cer<lat. vulg. nascëre (cf. M.-L., $\left.R E W, \mathrm{n}^{\circ} 5832\right)$; no $(28, \mathrm{~A}, 30)$ e o $(24, \mathrm{~B}, 45)$; no $(28, \mathrm{~A}, 58)$, nom $(28, \mathrm{~A}, 38)$ e não $(416, \mathrm{~A}, 50)<$ non; nemhum $(417, \mathrm{~A}, 18)$, nehũu $(27, \mathrm{~A}, 6)$ e nẽhũu $(26, \mathrm{~A}, 60)<$ nec ünu-; nẽguem $(30, \mathrm{~A}, 44)$ e ninguem $(417, \mathrm{~A}, 1)<$ nec quem, talvez com infl: do espanhol; nos $(75, \mathrm{~B}, 38)$ e os $(24, \mathrm{~A}, 69)$; nobre $(24, \mathrm{~B}, 54)$ e Nonbre (hũũa Nonbre [sic] capella, $(31, \mathrm{~B}, 31)<$ nobile(houve assimil. progressiva, se não se trata de lapso do escriba); noite $(28, \mathrm{~B}, 62) \mathrm{e}$ noute $(28, \mathrm{~A}, 2)$; oraçõõs $(78, \mathrm{~A}, 10)$ e oraçõees $(31, \mathrm{~A}, 2) ;$ Outrosy $(25, \mathrm{~B}, 62)$ e outross $\tilde{y}$ $(27, \mathrm{~B}, 34)$, de outro $<$ altëru- + si $<$ sic.

Padeceron $(410, \mathrm{~B}, 48)$ e padecerão $(408, \mathrm{~A}, 59$, no tít. do cap. II), ambas formas do perf. ( $3^{\mathrm{a}}$ p. pl.); padre $(25, \mathrm{~A}, 7)$ e pay $(29, \mathrm{~B}, 42)$; pallauras $(29, \mathrm{~B}, 23)$ e parauoas

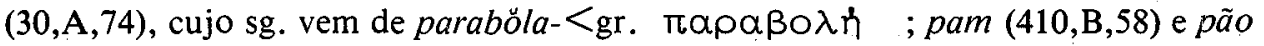
$(410, \mathrm{~B}, 52$, no tít. do cap. VIII<pane-; [peder] (pederas, 26,A,64) e [poder] (poder vos $h a, 417, \mathrm{~B}, 39)$, devido a assimil. o-e $>e-e$; pedir $(419, \mathrm{~A}, 28)$ e [pidir] (pidimos, $31, \mathrm{~B}, 46$ ) <lat. vulg. petĩre por pĕtĕre (as $\mathrm{f}$. com -di-devem-se à ass. $e-i>i-i$ ); peego $76, \mathrm{~A}, 44)$ e pego $(418, \mathrm{~B}, 63)<$ pèlăgu-; perfecto $(78, \mathrm{~B}, 55)$ e perfeito $(77, \mathrm{~B}, 23)$ $<$ perfectu-; perijgoo $(27, \mathrm{~A}, 2)$ e prjgoo $(76, \mathrm{~A}, 45)<$ pericŭlu- (a $2^{\mathrm{a}} \mathrm{f}$. vem da $1^{\text {a }}$ pela queda do e mudo pretónico); [perseguir] (persegujdos, 416, B,22) e [persiguir] (persiguirõ, 408,B,25)< lat. vulg. përsěquĕre, por persěqui $\left(\mathrm{a} 2^{\mathrm{a}} \mathrm{f}\right.$. vem da $1^{\mathrm{a}}$ por ass.: e-i>i-i [poboar] (poboada, 412,A,7), [poborar] (poborey, 29,B,39) e [povorar] (pouorou, 26,A,11)<pŏpüläre; pobre(s) $(24, \mathrm{~B}, 42)$, proue(s) $(411, \mathrm{~A}, 5) \mathrm{e}$ porue $(28, \mathrm{~B}, 29)<$ paupĕre-, através de *pöpëre (cf. Serafim da Silva Neto, Fontes 
do Latim Vulgar, p. 21); poer $(25, \mathrm{~A}, 62)$ e por $(409, \mathrm{~A}, 44$, no tít. do cap. IV)<pōnëre; pos $(24, \mathrm{~B}, 1)$ e pose (poseos em cruz, 27, $\mathrm{B}, 61)<p o ̋ s(u) i t$ (o -e final da f. port. mantinha-se quanto seguido de $l o$, la, etc.: pose-lo $>$ pose-o; cf. E.W., Fr. Lat., $\S 143,3, \mathrm{c})$; [pormeter] (pormeteo, 27,A,43) e [prometer] (prometi, 411,B,32)<prŏmittëre; prougue $(413, \mathrm{~A}, 55)$, prougueu $(78, \mathrm{~B}, 19)$ e prouve $(24, \mathrm{~A}, 69)<$ placuit $\left(\mathrm{a} 2^{\mathrm{a}}\right.$ f. é anal. com as dos v. regulares da $2^{\mathrm{a}}$ conj.); [preçar] (preçauã , 413,A,25) e [prezar] (prezauase, $75, \mathrm{~B}, 41)<$ pretiāre; procissão $(411, \mathrm{~A}, 33$ no tít. do cap. IX) e procisson $(411, \mathrm{~A}, 62)<$ processiōne-

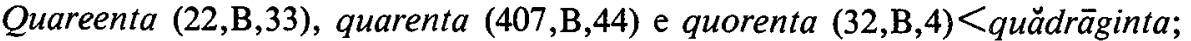
quis $(25, \mathrm{~A}, 50)$ e quisse $(410, \mathrm{~A}, 56)<*$ quaesit por quaesiit (o $i$ port. deve-se à metafonia, isto é, à infl. assimiladora do $i$ da des. lat. da $1^{\text {a }}$ p.s. quiseredes $(27, \mathrm{~A}, 68) \mathrm{e}$ quejserdes $(27, \mathrm{~A}, 32)<$ quaes(i)eritis; Regno $(31, \mathrm{~B}, 45)$ e reino $(416, \mathrm{~B}, 6)<$ regnu $(\mathrm{co}-$ mo se disse mais atrás, neste caso pode tratar-se apenas de duas maneiras de grafar a semivogal $i)$; rega $(78, \mathrm{~A}, 48)$ e regra $(76, \mathrm{~B}, 65)<$ régŭld̆-; Regnou $(22, \mathrm{~B}, 53)$ e Reynou $(32, \mathrm{~A}, 2)$ (veja-se o que dizemos quanto a reino); Relegiom $(78, \mathrm{~B}, 15)$ e Religiom $(76, \mathrm{~A}, 51)<$ rĕlĭgiōone-; Reueremça $(77, \mathrm{~A}, 37)$ e reueremçia $(418, \mathrm{~B}, 15)<$ rěverentiá; Rezam $(27, \mathrm{~A}, 29)$, rezão $(419, \mathrm{~B}, 41)$ e $\operatorname{Razom}(26, \mathrm{~B}, 10)<$ rătĭōne-.

Ssa $(27, \mathrm{~B}, 44)$, de sua $(22, \mathrm{~A}, 52)<$ süa- (por próclise); ssouberom $(26, \mathrm{~B}, 56)$ e souberão $(420, \mathrm{~B}, 6)$; ssabede $(28, \mathrm{~A}, 34)$ e sabee [2a p. pl.] $(76, \mathrm{~B}, 30)$; saçom $(27, \mathrm{~B}, 17)$ e ssazoim $(26, \mathrm{~B}, 9)<$ sătione-; saluação $(420, \mathrm{~B}, 5)$ e saluaçõ $(407, \mathrm{~A}, 34)<$ salvātiōone-; sancto $(407, \mathrm{~A}, 35)$, sam $(23, \mathrm{~A}, 6)$ e são $(418, \mathrm{~B}, 1)<$ sanctu- $\left(01^{\circ}\right.$ deu os outros dois

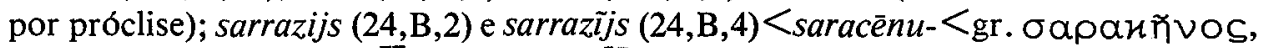
talvez com base ár. xarq $\ddot{i i n}$, pl. de xarqiī, '(muçulmano) oriental" (cf. J.P.M., Comentários a alguns arabismos do Dicionário de Nascentes, s. v. sarraceno, e Infl. $A r$., II, 227-237), e cruzamento com f. port. de or. ár. em -i, p. ex. arabi e maravedi; saseenta (22,B.42) e sesseenta $(24, A, 54)<$ sexāginta; segodo [sic] $(77, \mathrm{~A}, 15)$, em que o escriba se esqueceu de representar graficamente a nasalidade, e segundo $(24, \mathrm{~B}, 76)<$ sĕcŭndu-; formas do verbo ser $(417, \mathrm{~B}, 36)$ ou seer $(26, \mathrm{~A}, 59)<$ sĕdère: soom $(26, \mathrm{~B}, 49)$, sõo $(77, \mathrm{~B}, 69)$ e soo $(39, \mathrm{~A}, 16)<$ sum $\left(02^{\circ} \mathrm{da} 3^{\circ} \mathrm{f}\right.$. é anal. com o da $1^{\text {a }}$ p. sing. de outros verbos (cf. E.W., Fr. Lat., * 198, 3); $\operatorname{som}(78, \mathrm{~A}, 21)$ e são $(417, \mathrm{~B}, 37)<$ sŭnt (a $2^{\mathrm{a}} \mathrm{f}$. é por anal. com cão, dão, hão e estão); erom $(77, \mathrm{~B}, 21) \mathrm{e}$ erã $(408, \mathrm{~A}, 41)$; foram $(411, \mathrm{~A}, 46)$ e forom $(418, \mathrm{~A}, 8)$; fosse $(408, \mathrm{~A}, 49)$ e ffoisse $(28, \mathrm{~B}, 67)<f u ̈ u s s e t$, ou antes, $*$ füsset, como se vê das restantes f. do tempo, quer em port., quer nas outras línguas românicas (o $i$ deve-se a confusão com foi-se); seeria $(30, \mathrm{~A}, 71)$ e seria $(28, \mathrm{~A}, 8)$; seemdo $(25, \mathrm{~B}, 50)$ e sendo $(417, \mathrm{~B}, 30)<$ sedendo; semelhauel $(26, \mathrm{~A}, 49)$ e semelhauil $(413, \mathrm{~A}, 16)$, deriv. de semelhar *similiāre, por similāre (cf. J.P.M., D.E., p. 1965); se não $(418, \mathrm{~A}, 2)$ e ssenom $(27, \mathrm{~A}, 47)$, de se +nom ou<si +non; sepultado $(22, \mathrm{~A}, 30)$ e sopultado, isto é, supultado $(30, \mathrm{~B}, 59)$, forma em que houve ass. do $e$ ao $p$ (labialização) e ao $u$ (cf. Huber, Alpt., * 260, e E.W., Fr. lat., * 41,5); sepultura (408,A,59, no tít. do cap. II), e supultura $(78, \mathrm{~A}, 44)$, f. que tem a mesma explicação que supultado; so $(25, \mathrm{~A}, 24)$ e $s u(414, \mathrm{~B}, 2)<s u b$; sobçessores $(23, \mathrm{~B}, 9)$, pseudolatinismo ortogr., por ultracorrecção, e sucessores $(412, \mathrm{~B}, 7)<$ successōres; sometida $(s)(78, B, 44)$ e sumitida $(414, \mathrm{~B}, 41)$, formas refeitas. 
Tã $(25, \mathrm{~A}, 54)$ e tão $(417, \mathrm{~A}, 51)<$ tam; formas do verbo [te(e)r]: teerẽ $(408, \mathrm{~A}$, $53)$ e terem $(416, \mathrm{~B}, 45)$; teem $(31, \mathrm{~A}, 1)$ e tem $(?)(417, \mathrm{~B}, 55)<$ tènént; tijnha $(29, \mathrm{~A}, 11)$ e tinha $(418, \mathrm{~B}, 42)<$ ténébat; tevera $(25, \mathrm{~B}, 41)$ e tivera $(419, \mathrm{~A}, 13)$; teendo $(26, \mathrm{~B}, 69) \mathrm{e}$ tendo $(416, \mathrm{~A}, 45)<$ tënendo; templo $(25, \mathrm{~A}, 52)$ e tempro $(77, \mathrm{~B}, 29)<$ templu-; terceira $(410, \mathrm{~B}, 26)$ e treçeiro $(28, \mathrm{~B}, 70)<$ tertiariu-, com metáteses normais; termno $(411, \mathrm{~B}, 39)$ e termo(s) $(416, \mathrm{~B}, 41)<$ terminu-; todo $(23, \mathrm{~B}, 8)$ e tudo $(417, \mathrm{~B}, 2)<$ tötu-; formas do verbo trager $(412, \mathrm{~B}, 2)$ ou trazer $(77, \mathrm{~A}, 42)<*$ trăgëre ou *tracere, por trăhëre (cf. J. P.M., D.E., s.v. trazer): tragedes $(413, \mathrm{~B}, 10)<*$ tragètis e trazedes $(28, \mathrm{~A}, 45)$; tragia $(408, \mathrm{~A}, 8)$ e trazia $(413, \mathrm{~A}, 41$, no tít. do cap. XIII) <*trage $\bar{e}(b) a t$ e ${ }^{*}$ tracēe(b)at); tragiam $(409, \mathrm{~B}, 14)<*$ tragê(b)ant e traziom $(418, \mathrm{~A}, 50) ;$ trouue $(31, \mathrm{~A}, 57)$, por anal. com houve; trouxe $(25, \mathrm{~B}, 8)$ e trouxi $(78, \mathrm{~B}, 56)<*$ traxuit; trouuerom $(418, \mathrm{~B}, 7)$, por anal. com houvérom, e trouxerom $(416, \mathrm{~A}, 14) ;$ tragendo $(408, \mathrm{~B}, 18)<*$ tragendo e trazendo $(416, \mathrm{~A}, 26)$; trigo $(24, \mathrm{~B}, 44)$ e triigo $(411, \mathrm{~A}$, $16)<$ triticu; trijnta $(22, \mathrm{~A}, 50)$ e trinta $(22, \mathrm{~B}, 43)<$ triginta (a evolução foi: triginta $>$ trienta $>*$ treinta $>$ triinta $>$ trinta; cf. Neto, Fontes, p. 93).

Hum $(409, \mathrm{~A}, 44$, no tít. do cap. IV) e hũu $(22, \mathrm{~A}, 26)<\bar{u} n u-$; hums $(416, \mathrm{~B}, 23)$ e hũũs $(78, \mathrm{~A}, 45)<$ ūnos; vigilia $(76, \mathrm{~B}, 40)$ e vela $(28, \mathrm{~B}, 63)$; veendo $(28, \mathrm{~A}, 8)$ e Vemdo $(416, \mathrm{~B}, 22)<$ videndo; vjnte $(31, \mathrm{~B}, 57)$ e vijnte $(22, \mathrm{~A}, 21)<v i g i n t i$ (a evolução foi: vigint $i>v i g e n t i>v i e n t i>v i i n t i>v e i n t e>v i i n t e>v i n t e ;$ of. Neto, Fontes, $p .93) ;$ uestiduras $\left(28, \mathrm{~A}, 2^{\circ} 24\right)$ e uistiduras $(28, \mathrm{~B}, 23)$ vestituras (ass. $e-i>i-i$; cf. Huber, Altp., § 259); formas do verbo vyr $(417, \mathrm{~A}, 14)$ ou vijr $(26, \mathrm{~B}, 34)<$ vènïre: veem $(26, \mathrm{~A}, 24)$ e vem $(417, \mathrm{~B}, 65)<*$ venent por ventunt; uiinham $(409, \mathrm{~B}, 36)$ e vinham $(27, \mathrm{~B}, 51)<*$ veniant, por venibant, de veniēbant 4 ; uẽẽo $(408, \mathrm{~A}, 3)$, ueo $(29, \mathrm{~A}, 58) \mathrm{e}$ veyo $(416, \mathrm{~A}, 45)<v e \bar{n} i t$ (a vog. - $o$ deve-sé à anal. com os pretéritos fracos); ueerom $(26, \mathrm{~A}, 30)$ e vieram $(24, \mathrm{~B}, 1)<$ vènērunt; ueera $(31, \mathrm{~B}, 51)$ e viera $(417, \mathrm{~B}, 4)<$ vènèrat, com deslocação do acento; neesse $(30, \mathrm{~A}, 72)$ e uiesse $(22, \mathrm{~B}, 51)<*$ venesset, por venisset; vĩjdo $(27, \mathrm{~A}, 9)$ e vĩdo $(28, \mathrm{~A}, 48)<*$ veñ̄tu-, por ventu-; uertude $(31, \mathrm{~A}, 1-2) \mathrm{e}$ virtude $(24, \mathrm{~B}, 56)<$ virtūte-; uertuossa $(31, \mathrm{~A}, 50)$ e virtuosa $(24, \mathrm{~B}, 55)<$ virtuōsa-, documentado em Euquério (cf. A.N., D.E., s.v. virtuoso); visão (410,A,52 no tít. do cap. VII) e uisson $(410, \mathrm{~B}, 22)<$ visiōne-; voontade $(76, \mathrm{~B}, 73)$ e vontade $(416, \mathrm{~A}$, 45) $<$ voluntāte-.

Obs. final - É evidente que as formas divergentes aduzidas são-no, de facto, foneticamente e não meras variantes ortográficas.

Povzetek

\section{ZNAČILNOSTI BESEDIŠČA IN SEMANTIKE STARE PORTUGALŠČINE}

Stare portugalske kronike, kot jih imamo zbrane v Portugaliae Monumenta Historica, kažejo dokaj svojsko grafijo, različno celo od sočasnih knjižnih del. Za pravilno umevanje grafije je treba seveda upoštevati težo latinske tradicije, včasih imamo opraviti celo s psevdolatinizmi. Prepričljivi primeri pa vendar dokazujejo, da različen zapis ni zgolj ortografska varianta: gre za drugačno glasovno podobo neke besede. 